INPLASY

PROTOCOL

To cite: Chen et al. Effects of meditation on cancer pain symptoms: A systematic review and meta-analysis. Inplasy protocol 2020110018. doi:

10.37766/inplasy2020.11.0018

Received: 05 November 2020

Published: 05 November 2020

Corresponding author: Chen Yukun

tumorchen@163.com

Author Affiliation:

University of Traditional

Chinese Medicine

Support: There is no fund support.

Review Stage at time of this submission: The review has not yet started.

Conflicts of interest:

No potential conflict of interest.

\section{Effects of meditation on cancer pain symptoms: A systematic review and meta-analysis}

Chen, YK¹; Geng, SH²; Wang, XF³ Jia, LJ4.

Review question / Objective: Cancer pain is one of the most common complications of malignant tumors. Because of the side effects of opioids and other painkillers, cancer patients begin to seek meditation as an adjuvant therapy to relieve cancer pain. At present, although there are many studies on the enthusiasm of meditation in cancer treatment, most of them are limited to relieve depression, anxiety and other psychological symptoms of cancer patients, and it is controversial whether cancer pain can be treated. Therefore, we conducted a systematic review and meta-analysis of randomized controlled trials to evaluate the effect of meditation on cancer pain relief.

Condition being studied: Cancer pain symptoms.

INPLASY registration number: This protocol was registered with the International Platform of Registered Systematic Review and Meta-Analysis Protocols (INPLASY) on 05 November 2020 and was last updated on 05 November 2020 (registration number INPLASY2020110018).

\section{INTRODUCTION}

Review question / Objective: Cancer pain is one of the most common complications of malignant tumors. Because of the side effects of opioids and other painkillers, cancer patients begin to seek meditation as an adjuvant therapy to relieve cancer pain. At present, although there are many studies on the enthusiasm of meditation in cancer treatment, most of them are limited to relieve depression, anxiety and other psychological symptoms of cancer patients, and it is controversial whether cancer pain can be treated. Therefore, we conducted a systematic review and meta- 
analysis of randomized controlled trials to evaluate the effect of meditation on cancer pain relief

Condition being studied: Cancer pain symptoms.

\section{METHODS}

Participant or population: Patients with cancer pain.

Intervention: Meditation as an adjuvant therapy to relieve cancer pain.

\section{Comparator: conventional nursing treatment.}

Study designs to be included: Randomized controlled trials.

Eligibility criteria: We believe that if the study is a randomized clinical trial for cancer pain patients that compares meditation with another treatment strategy, and the intervention time is at least one week, then we consider these studies to meet the inclusion criteria. The exclusion criteria are as follows: (1) Observational research and retrospective research (2) Non-English literature; (3) Not a psychosocial intervention related to mindfulness meditation; (4) The research object is not a cancer pain patient; (5) The intervention time is less than 1 week study; (6) There are no studies evaluating meditation therapy[20]. Two independent researchers (WXF, GSH) reviewed the title and abstract of the study, and searched for full-text evaluation studies that met the inclusion criteria. Two reviewers will reexamine all papers that have not been unanimously excluded or included until a consensus is reached. We extracted the following data from each selected study: total number of subjects, sex, trial time, difference in pain degree before and after treatment (mean [SD]), difference in quality of life before and after treatment (mean [SD]) and difference in fatigue degree before and after treatment (mean [SD]). To ensure consistency, for studies that collect data at a series of time points, only the data after the last intervention is used to calculate the magnitude of the effect.

Information sources: This systematic review and meta-analysis is reported in accordance with the Preferred Reporting Project (PRISMA) statement for systematic reviews and meta-analysis[19]. We performed a comprehensive literature search in PubMed, Mentalinfo, Cochrane and Embase databases. Combine keywords related to cancer (for example, tumor or cancer) with keywords related to intervention (for example, meditation or mindfulness or mindfulness-based stress (MBSR) or yoga) and keywords related to (pain or pain). Previously posted meditation comments on stress and other health issues have also been carefully screened until July 2020. Only includes research published in English with full text.

Main outcome(s): Degree of pain, quality of life, degree of fatigue.

Quality assessment / Risk of bias analysis: Jadad score.

Strategy of data synthesis: We searched PubMed, EMBASE, Cochrane and other major databases comprehensively. The key words searched were related to various types of meditation and cancer pain. Randomized controlled trials (Released by July 2020) were conducted to compare meditation therapy with conventional nursing treatment. The main evaluation indicators were cancer pain degree, quality of life and fatigue. Stata16.0 was used for meta-analysis.

Subgroup analysis: none.

Sensibility analysis: none.

Country(ies) involved: China.

Keywords: Meditation, cancer, pain.

Contributions of each author:

Author 1 - Chen YunKun.

Author 2 - Geng ShaoHui.

Author 3 - Wang XiaoFan.

Author 4 - Jia LiJun. 